\title{
Thickness Dependent Physical Properties of Thermally Evaporated Nanocrystalline CdSe Thin Films
}

\author{
Anuradha Purohit ${ }^{1} \cdot$ Subhash Chander $^{1} \cdot$ Satya Pal Nehra ${ }^{2,3} \cdot$ Mahendra Singh Dhaka $^{1,3}$
}

Received: 31 March 2015/Revised: 19 September 2015/Published online: 12 October 2015

(C) The Chinese Society for Metals and Springer-Verlag Berlin Heidelberg 2015

\begin{abstract}
This paper presents a study on thickness dependent physical properties of cadmium selenide thin films. The films of thickness 445, 631 and $810 \mathrm{~nm}$ were deposited employing thermal evaporation technique on glass and ITO-coated glass substrates followed by thermal annealing in air atmosphere at $200{ }^{\circ} \mathrm{C}$. These films were subjected to X-ray diffractometer, UV-Vis spectrophotometer, scanning electron microscopy (SEM) and electrometer for structural, optical, surface morphological and electrical analysis respectively. The structural analysis reveals that the films are nanocrystalline in nature with cubic phase and preferred orientation (111). The crystallographic parameters such as lattice constant, interplanar spacing, grain size, internal strain, dislocation density, number of crystallites per unit area and texture coefficient are calculated and discussed. The optical band gap is found in the range 1.75-1.92 eV and observed to increase with thickness. The SEM study shows that the annealed films are uniform, fully covered and well defined. The electrical analysis shows that the conductivity is varied with film thickness and found within the order of semiconductor behavior.
\end{abstract}

KEY WORDS: Thin films; X-ray diffraction; Optical properties; Electrical properties; Evaporation

\section{Introduction}

The compound semiconductors of II-VI group are extremely vibrant due to their potential uses in the solar cell and optoelectronic applications. Among these compound semiconductors, CdSe is encountered substantial attention due to its suitable optical band gap $1.74 \mathrm{eV}$ and high absorption coefficient. It is one of the most promising

Available online at http://link.springer.com/journal/40195

Subhash Chander

sckhurdra@gmail.com

1 Department of Physics, Mohanlal Sukhadia University, Udaipur 313001, India

2 Centre of Excellence for Energy and Environmental Studies, Deenbandhu Chhotu Ram University of Science and Technology, Murthal, Sonepat 131039, India

3 Microelectronics Research Center, Iowa State University of Science and Technology, Ames, IA 50011, USA n-type materials and used to produce photo-electronic devices such as solar cells, light-emitting diodes, PEC cells. It has cubic zinc blende and hexagonal wurtzite structures [1-6]. The quality of CdSe films is strongly dependent on deposition conditions, substrate temperature, substrate, annealing, doping and vacuum. A number of deposition techniques have been used to deposit CdSe thin films like electrodeposition, thermal evaporation, chemical bath deposition, pulsed laser deposition [7-12]. Among all deposition techniques, thermal evaporation technique is found most promising technique due to its high deposition rate, low consumption of material and economical way of deposition process. It is a physical vapor deposition technique which is based on evaporation of the material in vacuum by thermal energy and allowing the vapor stream of the charge to condense on the substrate to form a continuous film [13]. The deposition process is conducted under high vacuum to grow uniform and homogeneous thin films and to avoid oxide layers as well as impurities. A number of researchers have been worked on different 
growth conditions and structural analysis of CdSe thin films for optoelectronic device applications [14-18]. Kale and Lokhande [19] studied the effect of air annealing on the physical properties of chemically deposited CdSe nanocrystallites and found that the crystallinity was increased with air annealing. Basheer et al. [20] reported physical and luminescence properties of indium-doped CdSe films employing electron beam evaporation technique. Zhao et al. [21] deposited nanocrystalline CdSe thin films using chemical bath deposition. They found that the CdSe thin films showed cubic zinc blende structure and optical band gap was found in the range $1.80-1.90 \mathrm{eV}$. Recently, the effect of thickness and thermal annealing on the physical properties of CdSe thin films was carried out by Purohit et al. [22-24]. The thickness of thin films affects the physical properties viz. structural, electrical and optical properties. Generally, the crystallinity of a thin film is increased with thickness due to enlargement of grain size while energy band gap is decreased. Exceptionally, the crystallinity of thin films may be decreased with increasing thickness and energy band gap may be increased owing to the stacking of the smaller grains on the surface of the larger grains. Consequently, the structural and optical parameters as well as electrical properties may also be changed.

Though literature survey reveals that the work on large scale has done on CdSe thin films yet the thickness dependent study on physical properties of the thermally annealed films at $200{ }^{\circ} \mathrm{C}$ is not well understood. Therefore, the present work is aimed to carry out and enhance the thickness dependent physical properties of CdSe thin films employing thermal evaporation method. The structural, optical, surface morphological and electrical properties are measured using XRD, UV-Vis spectrophotometer, SEM and electrometer respectively. The crystallographic and optical parameters are also calculated and discussed in detail.

\section{Experimental Details}

CdSe thin films were grown on chemically cleaned glass and ITO-coated glass substrates employing thermal evaporation technique. The glass substrates were used to carry out structural, optical and surface morphological properties while ITO-coated glass substrates for electrical properties. CdSe powder of purity more than $99.99 \%$ was procured from Alfa Aesar and used as source material in pellet form. The substrates cleaning plays a crucial role during the deposition process and therefore these substrates were immersed into the distilled water then acetone and followed by ethyl alcohol. The dimensions of substrates was $10 \mathrm{~mm} \times 10 \mathrm{~mm} \times 1 \mathrm{~mm}$. In the thermal evaporation technique, tantalum, molybdenum and tungsten boats may be used. In this study, molybdenum boat was used inside the chamber to keep the CdSe pellet. The vacuum coating chamber was evacuated up to a pressure $1.33 \times 10^{-4} \mathrm{~Pa}$ using rotary and diffusion pumps. The thin films of different thickness were deposited followed by annealing in air atmosphere at temperature $200{ }^{\circ} \mathrm{C}$ in a furnace for $1 \mathrm{~h}$. The thickness was verified by ellipsometry (Woolam M2000) and found 445, 631 and $810 \mathrm{~nm}$. The structural properties of annealed thin films were measured using $\mathrm{X}$-ray diffractometer (Bruker AXS D8 Advance) of $\mathrm{Cu} K_{\alpha}$ radiation in the $2 \theta$ range from $20^{\circ}$ to $60^{\circ}$. The UV-Vis spectrophotometer (SHIMADZU UV-2450) was used to investigate the optical properties within the wavelength range $200-800 \mathrm{~nm}$. The surface morphological study was carried out using scanning electron microscopy (Zeiss EVO 18 ) and the electrical analysis of these films was undertaken employing an electrometer (Agilent B2901A).

\section{Results and Discussion}

\subsection{Structural Analysis}

The X-ray diffraction pattern of thermally evaporated CdSe thin films is presented in Fig. 1.

The XRD pattern of the film of thickness $445 \mathrm{~nm}$ shows sharp and intense peak corresponding to reflection (111) at angular position $25.42^{\circ}$ which is the preferred reflection and well agreed with the standard JCPDS data file (652891) [25]. The angular position of the preferred peak (111) is observed to be shifted toward higher side with thickness owing to variation in the corresponding lattice

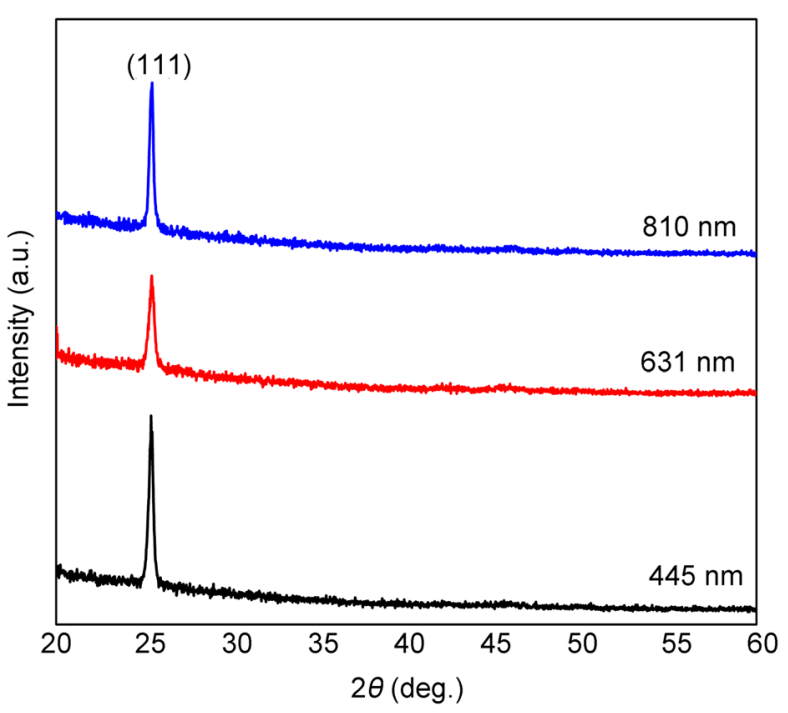

Fig. 1 XRD patterns of CdSe thin films 
constant. The peak intensity is decreased for thickness $631 \mathrm{~nm}$ and increased for $810 \mathrm{~nm}$ which revealed to the variation in the crystallinity [26]. The crystallographic parameters such as lattice constant, inter-planar spacing, grain size, internal strain, dislocation density, number of crystallites per unit area and texture coefficient were calculated using relation concerned [23]. The inter-planar spacing $(d)$, lattice constant $(a)$ and number of crystallites per unit area $(N)$ are tabulated in Table 1.

The inter-planar spacing and lattice constant are found to slightly decrease with film thickness corresponding to prominent reflection (111) which may be attributed to the shift of angular position toward higher side (Table 1). The number of crystallites per unit area $(N)$ is varied in the range $(20.3-46.1) \times 10^{15} / \mathrm{m}^{2}$ with thickness and observed to increase for thickness $631 \mathrm{~nm}$ while decrease for $810 \mathrm{~nm}$ owing to variation in corresponding grain size. The grain size $(D)$ was calculated using Scherrer formula and observed to vary in the range $23.90-34.03 \mathrm{~nm}$ with thickness (Fig. 2a). It is decreased for thickness $631 \mathrm{~nm}$ which may be attributed to the formation of new grains on the surface of larger grains and increment in corresponding full width at half maxima [27]. The grain size is observed to increase for $810 \mathrm{~nm}$ which may be attributed to increment in nucleus size leading to larger clusters owing to the formation of continuous films [13]. The dislocation in a film may be arisen due to slight rotation of two islands with respect to each other as well as by the presence of grain boundaries. The internal strain is varied between 0.276 and 0.393 and found to be increased for thickness $631 \mathrm{~nm}$ while decreased for $810 \mathrm{~nm}$ due to variation in corresponding full width at half maxima (Fig. 2b). The increasing strain shows compressive nature while the decreasing strain shows tensile nature which may be attributed to the variation in crystallinity [28]. The dislocation density is observed to increase for thickness $631 \mathrm{~nm}$ while decrease for $810 \mathrm{~nm}$ owing to variation in corresponding crystallinity and grain size. The results are in agreement with earlier reported work of Velumani et al. [8] and Reddy et al. [26]. The texture is defined as a non-random distribution of crystal orientation [29] and texture coefficient (TC) presents the texture of a particular plane which implies the preferred growth along deviation from unity [30]. It was calculated using relation concerned and is shown in Fig. 3.
The texture coefficient is observed to be varied in the range 2.16-2.43 with film thickness corresponding to prominent plane (111) and found higher than one $(T C>1)$ which revealed that the films become more textured and all the grains are oriented in a specific direction of (111) orientation.

\subsection{Optical Analysis}

The absorbance and transmittance spectra of CdSe thin films were recorded in the wavelength range $200-800 \mathrm{~nm}$ employing UV-Vis spectrophotometer and are shown in Fig. 4.

It is observed in Fig. 4a that the absorbance is decreased with wavelength and found maximum at lower wavelength. The absorption edge is found to decrease for thickness $631 \mathrm{~nm}$ while increase for $810 \mathrm{~nm}$ which may be due to the discontinuity of grain boundary and increase in disorderness. The optical transmittance is observed to increase with wavelength and found more than $58 \%$ for thickness $445 \mathrm{~nm}$ and $810 \mathrm{~nm}$ as well as observed maximum $100 \%$ for thickness $631 \mathrm{~nm}$ in the visible range. The transmittance is found to increase with film thickness owing to dominance of scattering mechanism of grain boundaries. The optical band gap may be calculated using Tauc relation $(\alpha h v)^{2}$ versus hv [31]. The Tauc plot of the films is shown in Fig. 5 and found approximately linear which revealed to the direct transitions between valence band and conduction band. The optical energy band gap was evaluated by extrapolating the straight line of the plot corresponding to zero absorption coefficients and the intercepts were given the corresponding optical band gap.

The optical band gap of the films is varied in the range $1.75-1.92 \mathrm{eV}$ and found to increase with thickness which may be attributed to the variation in the crystallinity owing to the stacking of new smaller grains on the larger grains. The results are in agreement with the earlier reported work of Nair et al. [32]. The refractive index of films was calculated using Herve-Vandamme formula [33] and found in the range 2.74-2.82. It is observed to decrease with film thickness due to increase in corresponding optical band gap and variation in packing density of the films [23]. These results are well supported by earlier reported work of Velumani et al. [8]. The extinction coefficient $(k)$ was

Table 1 Crystallographic parameters of CdSe thin films of different thickness

\begin{tabular}{|c|c|c|c|c|c|c|}
\hline \multirow[t]{2}{*}{ Thickness (nm) } & \multirow[t]{2}{*}{$2 \theta\left({ }^{\circ}\right)$} & \multirow[t]{2}{*}{ (hkl) } & \multicolumn{2}{|l|}{$d(\AA)$} & \multirow[t]{2}{*}{$a(\AA)$} & \multirow[t]{2}{*}{$N \times 10^{15}\left(\mathrm{~m}^{-2}\right)$} \\
\hline & & & Obs. & SD & & \\
\hline 445 & 25.42 & (111) & 3.50 & 3.51 & 6.055 & 20.3 \\
\hline 631 & 25.46 & (111) & 3.495 & 3.51 & 6.046 & 46.1 \\
\hline 810 & 25.48 & (111) & 3.492 & 3.51 & 6.041 & 20.5 \\
\hline
\end{tabular}



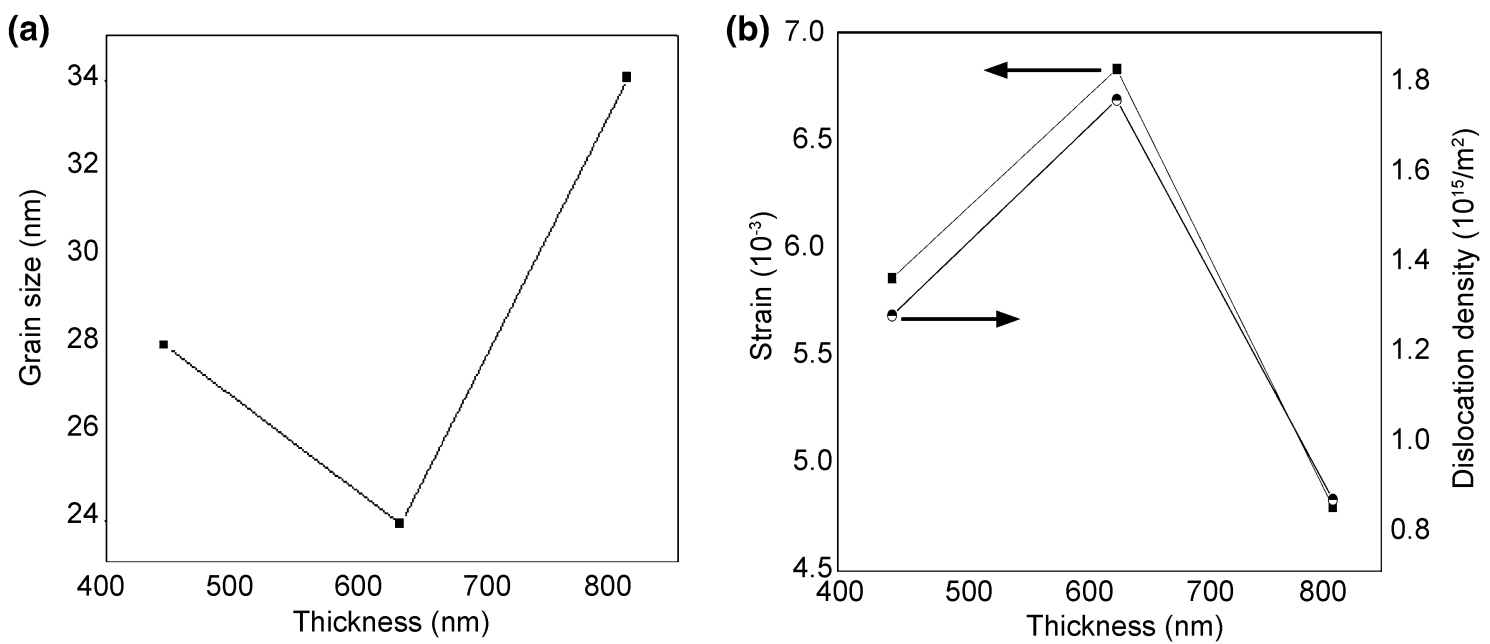

Fig. 2 Variation in grain size a, strain and dislocation density b with thickness of CdSe thin films

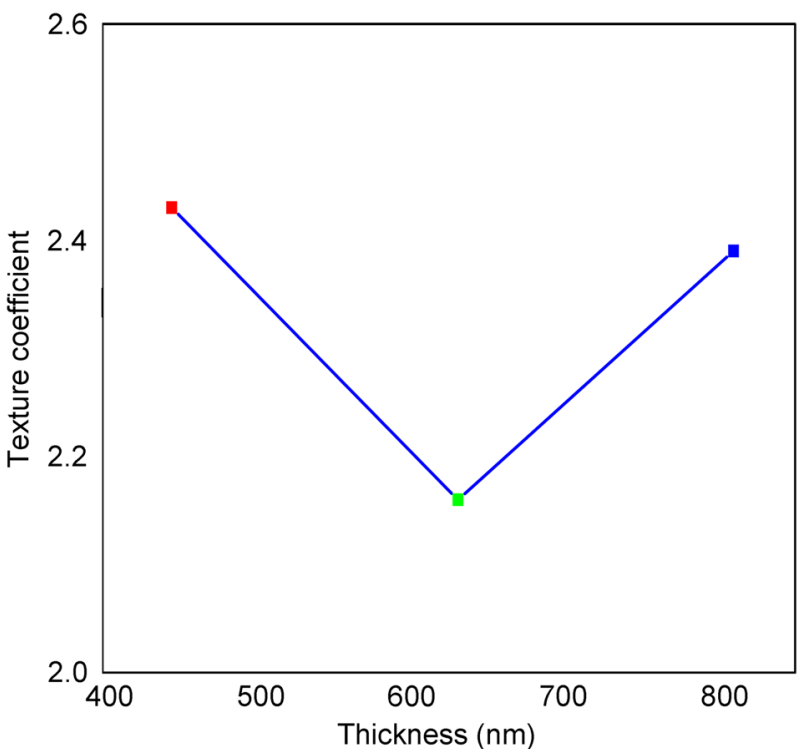

Fig. 3 Variation in texture coefficient with thickness of CdSe thin films

calculated using relation concerned [24] and shown in Fig. 5b. It is found in the order of $10^{-2}$ and observed to vary with thickness which revealed to the dominance of packing density of bound atoms [23].

\subsection{Surface Morphological Analysis}

The surface morphological properties of CdSe thin films were analyzed and SEM images are shown in Fig. 6. The SEM images of thermally evaporated CdSe thin films show that the films are homogeneous, fully covered and smooth. For thickness $445 \mathrm{~nm}$, the grains are distributed in similar in size, densely packed and well defined. Some crystal defects such as cracks and pin holes are also observed in film of thickness $445 \mathrm{~nm}$ which might be minimized or disappeared with film thickness. Some small spherical shape grains are observed to distribute over the substrate surface and the size of these grains is found to increase with thickness.

\subsection{Electrical Analysis}

The measurements of current-voltage characteristics of CdSe thin films were taken using an electrometer and are shown in Fig. 7. The variation in current with voltage for thermally evaporated CdSe thin films was found to be linear. Purohit et al. [23, 24] reported a similar currentvoltage behavior for $\mathrm{CdSe}$ thin films with film thickness and annealing temperature respectively. The electrical resistivity $(\rho)$ of the thin films was calculated using relation concerned [10] and found in the order of $10 \mathrm{ohm}-\mathrm{cm}$ as well as varied in the range (5.24-7.59) ohm-cm. The variation in resistivity may be attributed to the change in grain size and full width at half maxima owing to nanocrystalline nature of the films [20]. The conductivity of the films is found in the order of $10^{-1} \mathrm{mho} / \mathrm{cm}$ and varied in the range $(1.31-1.90) \times 10^{-1} \mathrm{mho} / \mathrm{cm}$. A similar behavior of resistivity and conductivity is also reported by Erat et al. [10] and Kale et al. [19].

\section{Conclusion}

The thickness dependent physical properties of CdSe thin films are reported in this paper and films of thickness 445, 631 and $810 \mathrm{~nm}$ were deposited employing thermal evaporation technique followed by thermal annealing at $200{ }^{\circ} \mathrm{C}$. The structural analysis showed that the films were 

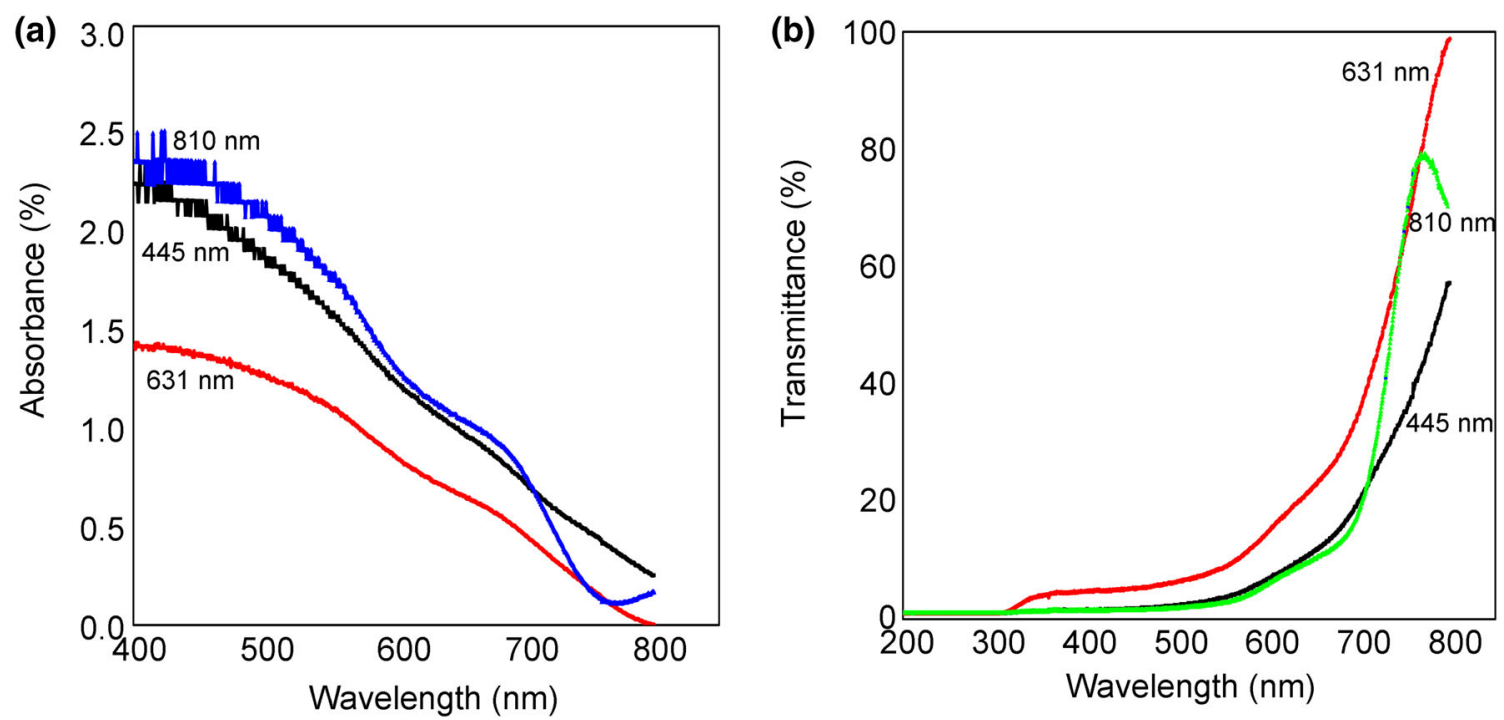

Fig. 4 Absorbance a and transmittance b optical spectra of CdSe thin films of different thickness
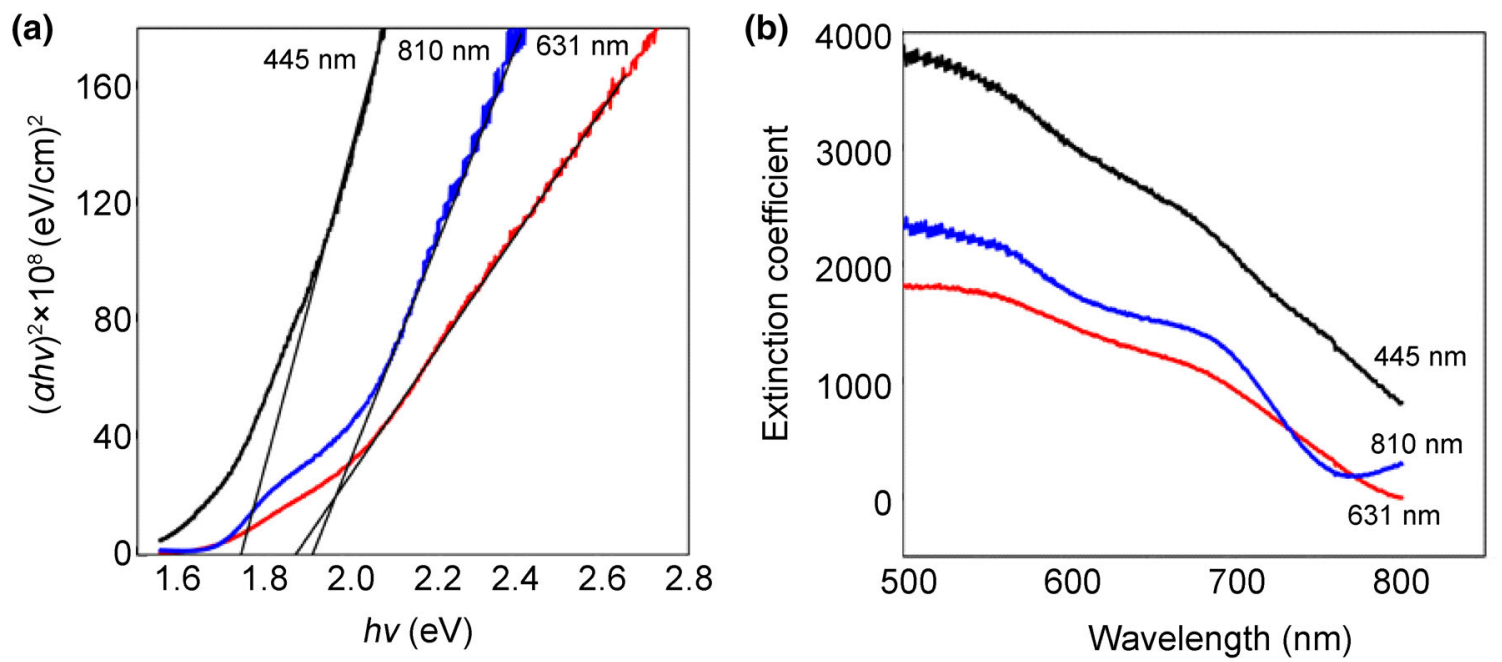

Fig. 5 Tauc plot $(\alpha h v)^{2}$ versus $h v$ a and extinction coefficient $(k) \mathbf{b}$ of CdSe thin films
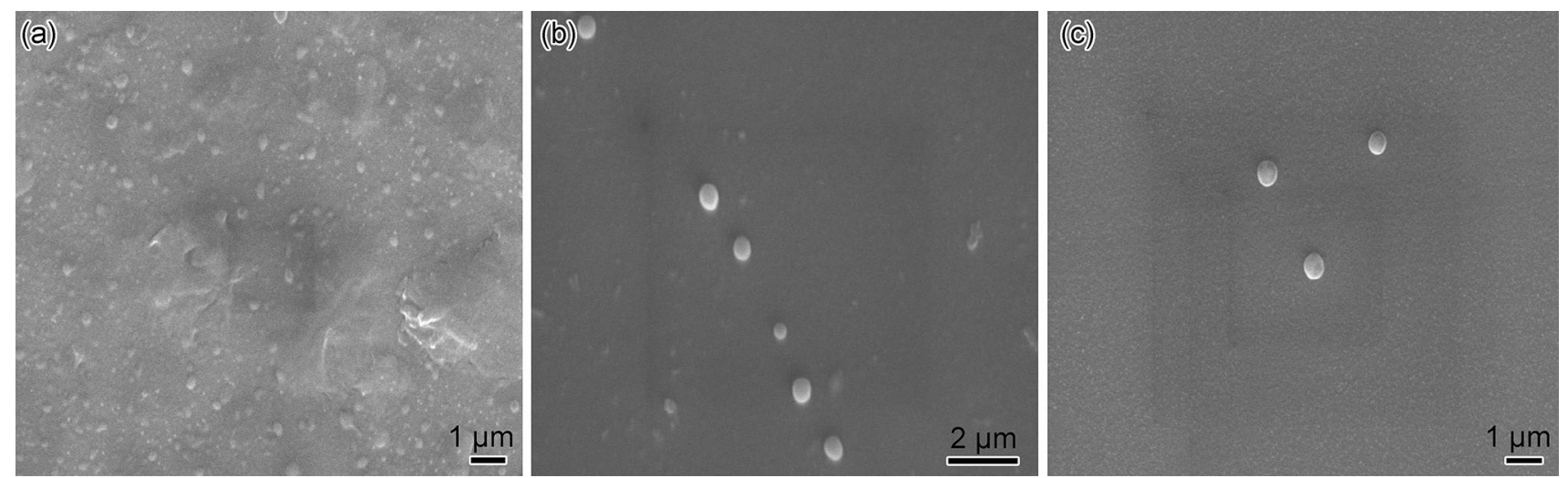

Fig. 6 SEM images of CdSe thin films of different thickness: a $445 \mathrm{~nm}$, b $631 \mathrm{~nm}$, c $810 \mathrm{~nm}$ 


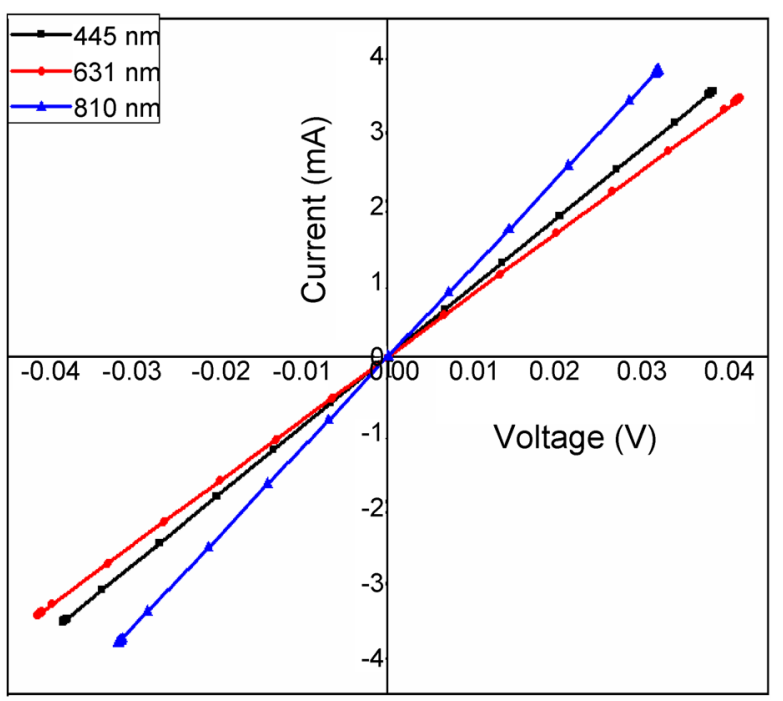

Fig. 7 Current-voltage characteristics of CdSe thin films of different thickness

nanocrystalline in nature with cubic phase having preferred orientation (111). The intensity of prominent peak was found to be increased with thickness owing to increase in the crystallinity. Despite the fact, the crystallinity of films of thickness $631 \mathrm{~nm}$ was found to decrease due to stacking of new smaller grains on the surface of larger grains. Consequently, the behavior of structural and optical parameters was changed and opposite behavior was observed for this thickness. The optical band gap was found in the range 1.75-1.92 eV and observed to increase with thickness. The SEM analysis showed that the annealed films were uniform, fully covered and well defined while some crystal defects such as cracks and pin holes were observed. The electrical analysis showed that the variation in current with voltage was found to be linear and resistivity was observed to vary with film thickness.

Acknowledgments The authors are thankful to the Centre for NonConventional Energy Resources, University of Rajasthan, Jaipur and Dr. Pawan Kulriya, Scientist, Inter University Acceleration Centre, New Delhi for film deposition and X-ray diffraction facility respectively. A.P. is grateful to the University Grants Commission, New Delhi for financial support under UGC-BSR fellowship No. F.25-1/ 2013-14(BSR)/7-123/2007(BSR).

\section{References}

[1] X. Zhang, S. Wang, D. Ding, X. Liu, J.H. Tan, J.K. Furdyna, Y.H. Zhang, D.J. Smith, J. Electron. Mater. 38, 1558 (2009)
[2] Y. Araki, K. Ohkuno, T. Furukawa, J. Saraie, J. Cryst. Growth 301-302, 809 (2007)

[3] W. Lee, W.C. Kwak, S.K. Min, J.C. Lee, W.S. Chae, Y.M. Sung, S.H. Han, Electrochem. Commun. 10, 1699 (2008)

[4] A. Kongkanand, K. Tvrdy, K. Takechi, M. Kuno, P.V. Kamat, J. Am. Chem. Soc. 130, 4007 (2008)

[5] J. Kaur, S.K. Tripathi, J. Alloys Compd. 622, 953 (2015)

[6] Y.M. Park, R. Andre, J. Kasprzak, L.S. Dang, E.B. Amalric, Appl. Surf. Sci. 253, 6946 (2007)

[7] C.M. Shen, X.G. Zhang, H.L. Li, Appl. Surf. Sci. 240, 34 (2005)

[8] S. Velumani, X. Mathew, P.J. Sebastian, S.K. Narayandass, D. Mangalaraj, Sol. Energy Mater. Sol. Cells 76, 347 (2003)

[9] A. Purohit, S. Chander, A. Nehra, S.P. Nehra, C. Lal, M.S. Dhaka, AIP Conf. Proc. 1661, 050009 (2015)

[10] S. Erat, H. Metin, M. Ari, Mater. Chem. Phys. 111, 114 (2008)

[11] M.A.H. Perez, J.A. Hernandez, G.C. Puente, J.R.V. Garcia, E.R. Salinas, Phys. E 40, 2535 (2008)

[12] R. Kumar, G. Kumar, A. Umar, J. Nanosci. Nanotechnol. 14, 1911 (2014)

[13] A. Goswami, Thin film fundamentals (New Age International Publishers, New Delhi, 2014)

[14] D.J. Miller, D. Hanemann, Sol. Energy Mater. 4, 223 (1981)

[15] R.A. Boudreau, R.D. Rauh, Sol. Energy Mater. 7, 385 (1982)

[16] M. Bouroushian, J.C. Got, Z. Loizos, N. Spyrellis, G. Maurin, Thin Solid Films 381, 39 (2001)

[17] P.P. Hankare, A.D. Jadhav, V.M. Bhuse, A.S. Khomane, K.M. Garadkar, Mater. Chem. Phys. 80, 102 (2003)

[18] P. Gaur, B.P. Malik, A. Gaur, Phys. B 457, 332 (2015)

[19] R.B. Kale, C.D. Lokhande, Appl. Surf. Sci. 223, 343 (2004)

[20] M.G.S.A. Basheer, K.S. Rajni, V.S. Vidhya, V. Swaminathan, A. Thayumanavan, K.R. Murali, M. Jayachandran, Cryst. Res. Technol. 46, 261 (2011)

[21] Y. Zhao, Z. Yan, J. Liu, A. Wei, Mater. Sci. Semicond. Process. 16, 1592 (2013)

[22] A. Purohit, S. Chander, A. Nehra, S.P. Nehra, C. Lal, M.S. Dhaka, AIP Conf. Proc. 1665, 080017 (2015)

[23] A. Purohit, S. Chander, S.P. Nehra, C. Lal, M.S. Dhaka, Opt. Mater. 47, 345 (2015)

[24] A. Purohit, S. Chander, S.P. Nehra, M.S. Dhaka, Phys. E 69, 342 (2015)

[25] Powder Diffraction Data File, Joint Committee of Powder Diffraction Standard, International Center for Diffraction Data, USA, Card No. 65-2891, 19-0191

[26] R.S. Reddy, A. Sreedhar, A.S. Reddy, S. Uthanna, Adv. Mater. Lett. 3, 239 (2012)

[27] S. Chander, M.S. Dhaka, Mater. Sci. Semicond. Proc. 40, 708 (2015)

[28] S.P. Nehra, S. Chander, A. Sharma, M.S. Dhaka, Mater. Sci. Semicond. Proc. 40, 26 (2015)

[29] K. Alamgir, T. Pervaiz, S. Arif, Energy Source A 34, 297 (2012)

[30] S.S. Shinde, P.S. Shinde, S.M. Pawar, A.V. Moholkar, C.H. Bhosale, K.Y. Rajpure, Solid State Sci. 10, 1209 (2008)

[31] M. Dhanam, R.R. Parbhu, P.K. Manoj, Mater. Chem. Phys. 107, 289 (2008)

[32] M.T.S. Nair, P.K. Nair, R.A. Zingaro, E.A. Meyers, J. Appl. Phys. 74, 1879 (1993)

[33] A. Purohit, S. Chander, A. Sharma, S.P. Nehra, M.S. Dhaka, Opt. Mater. 49, 51 (2015) 